\title{
High-temperature microwave effects in liquid media
}

\author{
Alexander Yu. Voloshko ${ }^{\mathrm{a}}$, Natalia A. Pinchukova ${ }^{\mathrm{a}}$, Oleg V. Shishkin, ${ }^{\mathrm{a}, \mathrm{b}}$ Valentin A.Chebanov $^{\mathrm{a}, \mathrm{b}}$ \\ a SSI "Institute for Single Crystals" of National Academy of Sciences of Ukraine, Lenin Ave., 60, \\ Kharkiv 61001, Ukraine \\ ${ }^{\mathrm{b}}$ V.N. Karazin Kharkiv National University, Svobody sq., 4, Kharkiv 61022, Ukraine
} pinchukova@isc.kharkov.com

Keywords: microwave irradiation, heating mode, non-linear interactions, high-boiling liquid, viscosity.

The heating behavior of high-boiling liquids under microwave (MW) irradiation was investigated. Linear and non-linear heating modes depending on the applied MW power level were observed. The non-linear interactions emerging at "high" power levels are ascribed to electromagnetic oscillations excitations resulting in non-linear heating speed growth and considerable decrease in energy input. The relationship between non-linearity degree and liquids' viscosity was established. The observed effects have been shown to apply not only to mere heating, but also to hightemperature chemical reactions. Future perspectives and practical applications of the work are also discussed.

\section{Introduction}

The sharp debate is still going on in the scientific community concerning the existence or non-existence of non-thermal microwave (MW) effects [1-6]. The benefits brought about by microwave irradiation some of the scientists ascribe to the totally thermal effects, arising from the dielectric heating mechanism, while the others incline towards the emergence of specific or non-thermal effects being the result of interactions between microwaves and matter, which are not directly connected to heating itself. Both groups of scientists give multiple proofs to substantiate their points of view; however, the discussion is not over yet $[7,8]$.

It is known from the theory of electromagnetic irradiation and dielectric media interactions that the linear dependence of the system parameters on the wave amplitude is observed if the wave energy is lower than the system's inner one [9].

In case when the wave energy exceeds the inner energy of the system non-linear interactions between electromagnetic field and medium occur.

In our previous investigation on the energy efficiency of microwave-assisted 
dehydration of orthophosphoric acid (OPA) the non-linearity in the consumed energy vs. MW power dependence was observed (Fig. 1) [10].

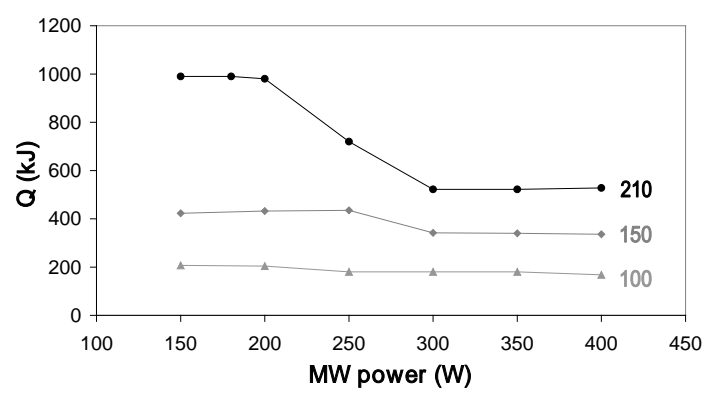

Figure 1. The total energy required for OPA dehydration at 100,150 , and $210^{\circ} \mathrm{C}$ [10].

This dependence could be divided into two ranges. The first range was characterized by relatively low power levels (up to $200 \mathrm{~W}$ under the certain experimental conditions), within which the process parameters change proportionally to the power, resulting in the constancy of the energy consumed irrespective of the power level. In this range the system's behavior could be mainly ascribed to the thermal effects of microwave irradiation. Meanwhile, gradual increase of microwave power resulted in the deviation from the energy constancy manifested by the slope of the curve that again plateaued at about $400 \mathrm{~W}$. Obviously, this slope was the evidence of the energy decrease in result of the emergence of specific microwave effects, which could also be defined as non-linear effects. This fact can be explained by that at "high" microwave powers the wave amplitude increases, and at some point where the wave energy becomes larger than the system's thermal energy, the process parameters change non-linearly with further increase in power level. Plateau on this dependence is obviously caused by the system's saturation and incapability of the dielectric to absorb more energy. Interestingly, that such non-linearity was detected only at high temperatures - above $150^{\circ} \mathrm{C}$.

Such observations stimulated us to conduct more careful study of microwave nonlinear effect with the use of other objects. To obtain unambiguous results and in purpose of simplicity of their interpretation the studied processes included heating only, with no chemical reactions involved. For this study we chose high-boiling organic liquids having different molecular weight and viscosity (glycerol, polyethyleneglycol (PEG-400), diethyleneglycol (DEG), as well as polyphosphoric acid (PPA), which were to be subjected to microwave heating in a wide range of the fixed MW power, and the energy consumed during MW heating was to be measured.

The main aim of the study was to prove experimentally the existence of the non-linear region in the energy vs. MW power dependence in the process of microwave heating of various liquids and to reveal the factors favoring and impeding non-linear interactions. Based on the results obtained with polyphosphoric acid dehydration, it can be assumed that the results of this study will be applicable to chemical 
processes as well, provided that they proceed under high temperatures.

\section{Results and discussion}

To demonstrate the influence of MW power on the energy consumption for MW heating of glycerol, PEG-400, DEG and PPA the appropriate plots were built (Fig. 2).

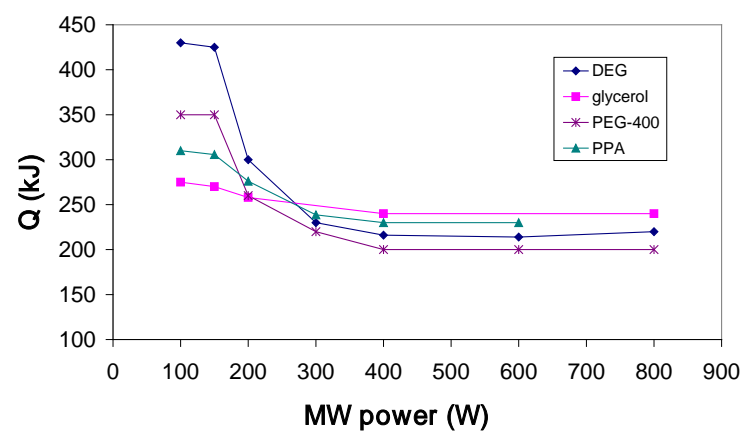

Figure 2. The total energy required for heating DEG, glycerol, PEG-400 and PPA in MW field to $200{ }^{\circ} \mathrm{C}$ vs. MW power input.

Fig. 2 shows that the energy consumption decreases with power increase for all the studied substances like in case of OPA [10]. The maximal difference between low- and high-power energy consumption was observed for DEG, while the minimal one for glycerol. PEG-400 and PPA demonstrated intermediate results. Interestingly, that decrease in the energy consumption was observed only for high temperatures $\left(200^{\circ} \mathrm{C}\right)$, meanwhile, for the low temperatures (below $150^{\circ} \mathrm{C}$ ) the constancy or slight variation of the energy consumption was observed throughout the whole range of the applied MW power levels in the experiments both on heating (Fig. 3 and 4) and on OPA dehydration (Fig. 1).

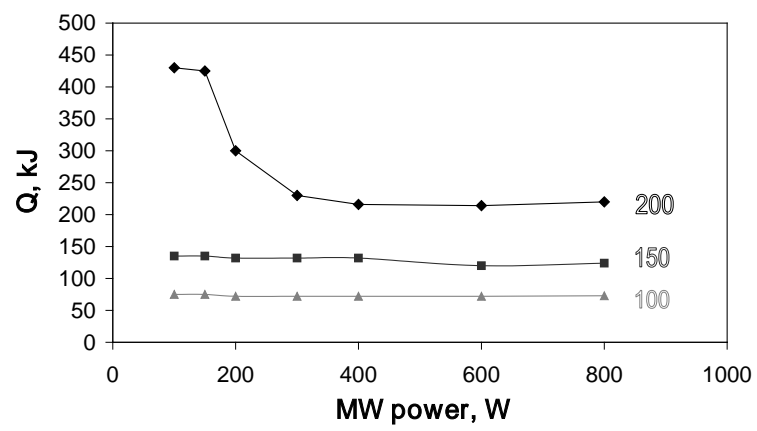

Figure 3. The total enery required for MW heating of DEG to 100,150 , and $200{ }^{\circ} \mathrm{C}$.

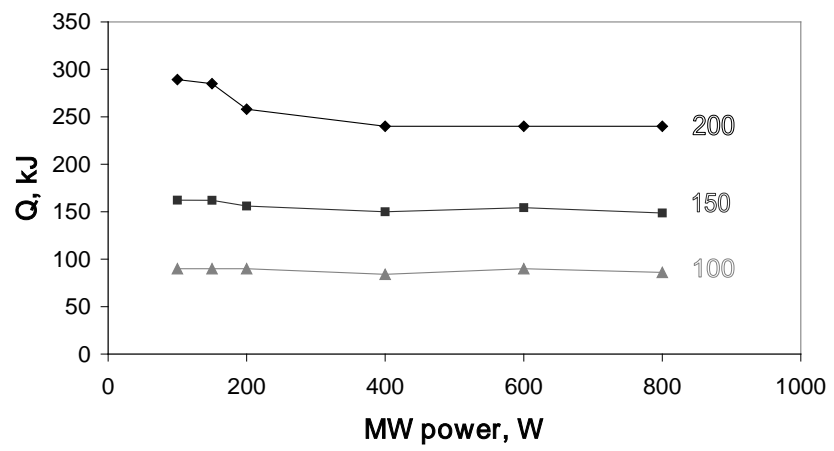

Figure 4. The total energy required for MW heating of glycerol to 100,150 , and $200{ }^{\circ} \mathrm{C}$.

Supposedly, this can be explained by the fact that at relatively low temperatures heating mode of highly boiling liquids is laminar and in the first approximation it can be assumed that the system's properties remain the same or change insignificantly with temperature rise. Thus, the heating behavior of the liquids in the low power range should not depend on the power value, i.e. should be similar to Joule's heating. Therefore, it is logical that the heating time is inversely proportional to the power 
value, resulting in the constancy of the energy consumption.

On the contrary, at high temperatures, especially close to the boiling point, heating acquires more turbulent mode and becomes more susceptible to the power applied. Presumably, the variation in the energy consumption for different powers, as demonstrated in Fig. 2, can be interpreted as the evidence of the specific microwave effects or non-linear interactions.

The following explanation may be offered. At low power values - in our case below $150 \mathrm{~W}$ - electric field strength or electromagnetic wave energy is lower than the thermal energy of the liquid, and this phase may be determined as a linear one. In other words, within this "low" power range MW heating mode of the liquids is similar to thermal heating and no or minor specific interactions, other than heating itself, take place between MW field and matter. With power increase the wave energy grows and at some point becomes equal to the thermal energy of the liquid. As follows from the experimental data (Fig. 2), this energy equality is reached at approximately $200 \mathrm{~W}$ which corresponds to the inflexion point in the curves. This value signifies the boundary between the linear and non-linear heating modes under the given experimental conditions. Below $200 \mathrm{~W}$ the energy introduced into the system is less than the system's heat energy and at $200 \mathrm{~W}$ they become even. The following are the energy estimations for heating DEG from 20 to $200^{\circ} \mathrm{C}$ :

$Q_{\text {heat }}=m \times c \times \Delta T=0.2 \times 2400 \times 180=86.4 \times 10^{3}$ $Q_{M W}=P \times t \times \eta=200 \times 1500 \times 0.3=90 \times 10^{3}$,

where $Q_{\text {heat }}$ is the heat energy ( $\mathrm{J}$ ), required for the liquid to be heated to the set temperature $T$ (K); $Q_{M W}$ is the energy of MW irradiation introduced into the system $(\mathrm{J}) ; \mathrm{m}$ is the weight of the sample (kg); $c$ is the liquid's specific heat capacity $\left(\mathrm{J} \cdot \mathrm{kg}^{-1} \cdot \mathrm{K}^{-1}\right)$; $P$ is MW power $(\mathrm{W})$; $t$ is the heating time (sec); and $\eta(0.3)$ is an efficiency factor obtained in our previous work [10] rounded to the first decimal digit.

It is evident from these estimations that the ratio Qmw/ Qheat is close to 1 when $\mathrm{MW}$ power is $200 \mathrm{~W}$, which confirms the above said.

In order to better illustrate how the heating behavior changes with MW power, heating speed (dT/dt) vs. MW power plots (Fig. 5 and 6) were built on the basis of the temperature profiles obtained when heating liquids at different MW power levels.

Mathematical treatment of the plot in Fig. 5 yielded three domains corresponding to three heating modes. The first one - up to 200 $\mathrm{W}$ - is related to the heating mode similar to Joule heating (heating speed is proportional to the power applied). The second domain between approx. 200 and 300 W - clearly indicates non-linearity described by the expression $y=x^{a}$, where $a>2$, however, to describe this dependence more precisely more routine studies are needed. It is in this MW 
power region that non-linear MW effects appear.

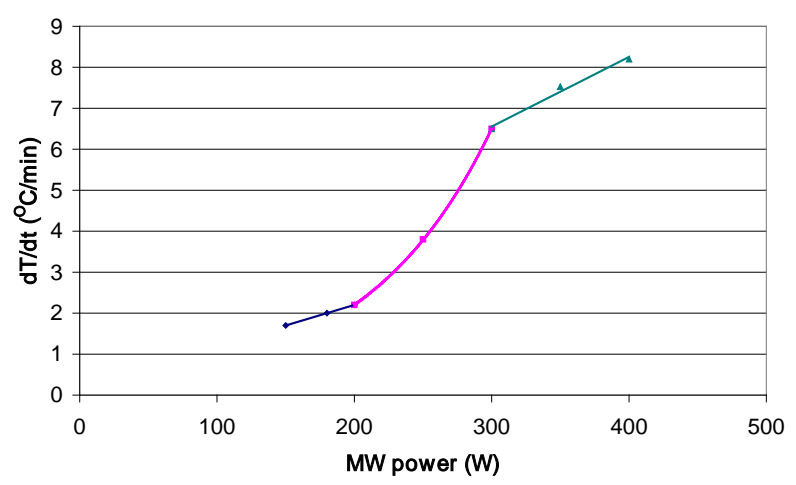

Fig. 5. OPA heating speed (dT/dt) vs. MW power plot, featuring three regions of heating mode: 1- linear (up to $200 \mathrm{~W}$ ), 2 - non-linear (200 to $300 \mathrm{~W}$ ), and 3 - linear (above $300 \mathrm{~W}$ ) (see explanations in the text).

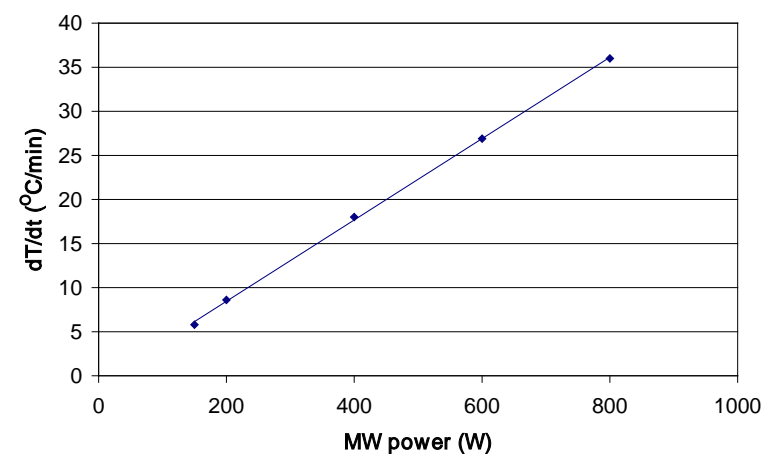

Fig. 6. Glycerol heating speed (dT/dt) vs. MW power plot, featuring linear dependence in the whole power range (see explanations in the text).

The non-linear behavior can be explained by that the system meets the condition when the wave energy exceeds the system's inner energy. The increments of non-linear interactions between the electromagnetic field and media result in the total energy drop (Fig. 1). The third domain is described by a linear equation again. Presumably, it can be explained by reaching the "saturation" state, which in our case means that the input MW power is equal to or higher than the dielectric's loss power (1) [11]:

$$
P=0.287 E^{2} f \varepsilon^{\prime \prime} \times 10^{-6} \text {, }
$$

where $P$ is dielectric's loss power $\left(\mathrm{W} / \mathrm{cm}^{3}\right) ; E$ is electric field strength $(\mathrm{V} / \mathrm{cm}) ; f$ is frequency $(\mathrm{MHz}) ; \varepsilon^{\prime \prime}$ is dielectric loss factor equal to $\varepsilon^{\prime} / \operatorname{tg} \delta$, in which $\varepsilon^{\prime}$ is dielectric permittivity, and $\operatorname{tg} \delta$ is dielectric loss tangent.

For example, resulting from equation (1), $\mathrm{P}$ value for $0.2 \mathrm{~kg}$ of DEG $\left(180 \mathrm{~cm}^{3}\right)$ amounts to ca $350 \mathrm{~W}$, which is in a good accordance with our experimental data (Fig. 3).

Thereby, it can be inferred that above some definite power, determined by the certain experimental conditions, no non-linear effects can progress any more. However, it should be noted that the differentiation between "low" and "high" power discussed in this paper only refer to the given experimental conditions (substance weight (0.2 kg), MW applicator model, flask geometry etc.) and the data given here cannot be directly applied to other systems without making necessary correlations.

However, it can be seen that the measure of non-linearity is not equal for different liquids, for some of them being pronounced (e.g. OPA, DEG) (Fig. 5), while for others approximating to the linear dependence within the whole power range (e.g. glicerol) (Fig. 6). Moreover, the differences between maximal and minimal energy consumption are not equal for different liquids either, being larger for the liquids exhibiting stronger non-linearity (cf. Fig. 3 and 
4). To find the explanation for the differences in heating behavior of the studied liquids and to compare them quantitatively, the energy decrease ratio (EDR) was calculated as the ratio of $\mathrm{Q}_{\max }$ corresponding to the highest energy consumed by the liquid when heated at $150 \mathrm{~W}$ to $\mathrm{Q}_{\min }$ corresponding to the lowest energy consumed at $400 \mathrm{~W}$ or above. It was shown that EDR values for different liquids vary considerably, reaching the highest value for DEG (2.0) and the lowest one for glycerol (1.1). It can be stated with certainty that the differences in EDR between the liquids are not related to the differences in their $\operatorname{tg} \delta$, as the latter does not depend on the power applied.

However, comparison of other physical properties of the liquids revealed definite relationship between EDR and liquid's viscosity, the most viscous liquid - glycerol having the lowest value of EDR, whereas the least viscous DEG having the highest one (Fig. 7).

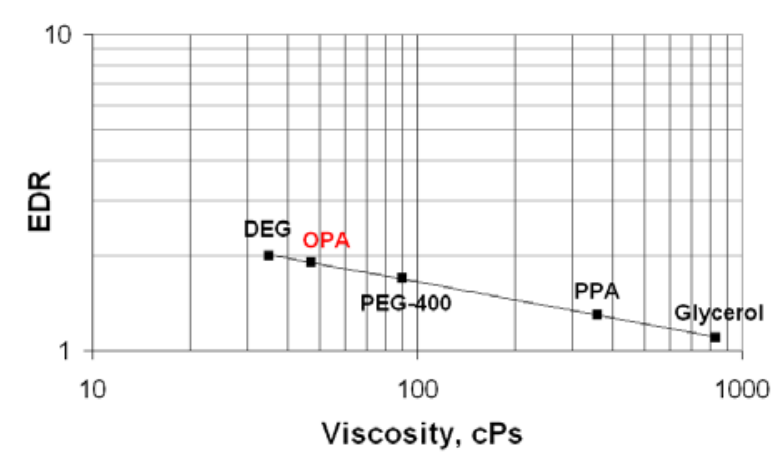

Fig. 7. Effect of dynamic viscosity of DEG, PEG-400, PPA, glycerol and OPA (for the latter the results are adopted from [10]) on the EDR (for $200{ }^{\circ} \mathrm{C}$ ).
This observation suggests that non-linear effects emerging in liquids heated by MW irradiation are directly related to their viscosity. Indeed, mathematical interpretation of the obtained dependence showed that the value of EDR is inversely proportional to $\eta^{1 / 3}$ that allows drawing some analogy with plasma electrodynamics. It is known that ionic viscosity of plasma suppresses excitation of oscillations, induced by alternating electric field [12, 13]. Thus, assuming EDR as a measure of nonlinearity in our case, it can be inferred that high viscosity of liquids also suppresses oscillations excitation upon MW heating, preventing nonlinear interactions between electromagnetic field and liquid media.

Fig. 7 also shows that OPA, though featuring a different kind of MW process, viz. dehydration reaction, in contrast to other liquids subjected to heating only, fits well the plot obtained for heating. This fact indicates that the obtained EDR vs. viscosity dependence is universal and, most likely, can be applied to the systems undergoing not only heating but other physical or chemical transformations accompanied by heating, provided that the basic experimental conditions (substance weight, MW equipment, apparatus etc.) are the same or very much alike. Otherwise, the results should be extrapolated as appropriate.

Interestingly, that the energy efficiency factor $(k)$ calculated as the ratio of the absorbed energy, estimated on the basis of thermal 
properties of the liquids, to the energy input determined experimentally ( $\mathrm{Q}_{\text {abs }} / \mathrm{Q}_{\text {input }}$ ) showed that the difference in $k$ was appreciable only at low powers, whereas at high powers, corresponding to plateau on Fig.1 and Fig. 2, this difference was negligible. For example, $k$ values calculated for DEG and glycerol, i. e. the liquids having the highest and the lowest EDR, at $200 \mathrm{~W}$ made 0.26 and 0.33 , respectively, whereas at $400 \mathrm{~W} k$ was found to be equal for both liquids (0.36).

On the basis of the obtained results it can be inferred that non-linear effects emerge in the system subjected to MW irradiation irrespective of the nature of the processes, and are dependent only on the medium temperature and MW power. It has also been shown that non-linear interactions between liquid medium and microwave irradiation of high intensity are suppressed by high viscosity of the former.

The results obtained are important for further investigation of MW-assisted processes. The findings of this work seem to be useful in the interpretation of the results obtained in the studies of MW-assisted processes, including chemical reactions, via non-linear interactions. In practical terms these results can be used in R\&D activities related to design and optimization of MW techniques. From scale-up point of view they can help in selecting right operational conditions for MW processes to achieve the utmost energy efficiency. The liquids viscosity should also be taken into account when choosing a solvent as a reaction medium, since the right choice of solvents can significantly improve MW processes outcome in terms of energy and time saving.

\section{Conclusions}

In result of this study it has been shown that microwave irradiation can induce nonlinear effects in liquids at high temperatures. At this, three ranges of interactions between microwave field and high-boiling liquids during their microwave processing (heating and dehydration reaction) at high temperatures $\left(>150^{\circ} \mathrm{C}\right.$ ) have been distinguished: linear, nonlinear and saturation ones.

It has been shown that non-linear interactions emerging in liquids are not dependant on the process type (physical or chemical transformation), but only on the media temperature and microwave power.

High viscosity of the liquids has been found to suppress non-linear interactions between microwaves and liquid media. This finding is in a good accordance with plasma physics theory, claiming that ionic viscosity impedes electromagnetic oscillations excitation, thus hampering non-linear interactions.

The data obtained in this work may be used in further fundamental researches on nonlinear effects in liquid media, as well as for practical purposes, e.g. in R\&D of novel microwave techniques. The findings of this work can undoubtedly be applied to microwave- 
assisted chemical reactions investigations, especially in the scope of "green” chemistry, chemical processes intensification and process scale-up.

Since this work can be considered a touchstone in the field of non-linear microwave effects investigation, further studies are needed aimed at mathematical treatment of the results obtained and development of a physical model describing high-temperature non-linear interactions between microwave irradiation and dielectric liquid media.

\section{Experimental part}

Glycerol (99 \%), PEG-400 (99 \%) and DEG (99 \%) used in the experiment were commercially available and chemically pure. PPA containing about $78 \%$ of $\mathrm{P}_{2} \mathrm{O}_{5}$ was prepared by means of OPA dehydration [10].

MW laboratory system MARS (CEM Corp., USA) operated at $2.45 \mathrm{GHz}$ and equipped with fiber-optic (FO) probe for temperature measurements was used in the experiments on MW heating. Magnetic stirrer was used for agitation. $200.0 \mathrm{~g}$ of the substance in the round-bottom flask were placed into the multimode MW cavity and heated to 100, 150, and $200{ }^{\circ} \mathrm{C}$ at the set input power. MW power was maintained at a constant level during the runs. Power and temperature profiles were continuously monitored via the computer connected to the MW device. When the set temperature was reached the MW oscillator switched off automatically and the total heating time was fixed. The total energy required for heating (Q) was calculated using the equation:

$$
Q=P \times t,
$$

where $P$ is $\mathrm{MW}$ power (W) generated by MW oscillator during the heating; $t$ is time required for heating (sec).

The liquid was then cooled down to the room temperature and heating procedure was repeated as described above for another power level.

Finally, for each liquid the series of heating runs was performed at different MW power levels in the range of 100 to $800 \mathrm{~W}$ with $50 \mathrm{~W}$ or $100 \mathrm{~W}$ step.

Each run was duplicated to ensure the repeatability of the results.

Dynamic viscosity of glycerol, PEG400, DEG, OPA and PPA used in the experiments was measured using Reolab-QC (Anton Paar, Austria).

\section{References}

[1] C.O. Kappe, A. Stadler, Microwaves in Organic and Medicinal Chemistry, WileyVCH: Weinheim 2006.

[2] Antonio de la Hoz, A. Diaz-Ortiz, A. Moreno, Microwaves in organic synthesis. Thermal and non-thermal microwave effects, Chem. Soc. Rev. 34 (2005) 164-178.

[3] A. Loupy, Microwaves in Organic Synthesis, 2nd ed., Wiley - VCH: Weinheim 2006. 
[4] M.A. Herrero, J.M. Kremsner, C.O. Kappe, [11] E.C. Okress, Microwave power Nonthermal Microwave Effects Revisited: On engineering. Volume 2. Applications, Academic the Importance of Internal Temperature Press, New York and London, 1968.

Monitoring and Agitation in Microwave [12] A.A. Rukhadze, V.P. Silin, Kinetic theory Chemistry, J. Org. Chem. 73 (2008) 36-47. of plasma drift-dissipative instability (in [5] J. Robinson, S. Kingman, D. Irvine, P. Russian), Uspehi Fizicheskih Nauk 96 (1968) Licence, A. Smith, G. Dimitrakis, D. 87-126.

Obermayer, C.O. Kappe, Understanding [13] V.S. Voitsenya, A.Yu. Voloshko, S.I. microwave heating effects in single mode type Solodovchenko, A.F. Shtan', Effect of ion cavities - theory and experiment, Phys. Chem. viscosity on shear stabilization of drift Chem. Phys. 12 (2010) 4750-4758.

[6] C. Antonio, R.T. Deam, Can "microwave dissipative instability, JETP Letters 26 (1977) effects" be explained by enhanced diffusion?, Phys. Chem. Chem. Phys. 9 (2007) 2976-2982.

[7] G.B. Dudley, A.E. Stiegman, M.R. Rosana, Correspondence on Microwave Effects in Organic Synthesis, Angew. Chem. Int. Ed. 52 (2013) 7918 - 7923.

[8] O.C. Kappe, Reply to the Correspondence on Microwave Effects in Organic Synthesis, Angew. Chem. Int. Ed. 52 (2013) 7924 - 7928.

[9] L.D. Landau, Y.M. Lifshitz, Electrodynamics of continua (Theoretical Physics, Vol. VIII), 4th ed., Physmatlit Moscow, 2003.

[10] N.A. Pinchukova, V.A. Chebanov, N.Y. Gorobets, L.V. Gudzenko, K.S. Ostras, O.V. Shishkin, L.A. Hulshof, A.Y. Voloshko, Beneficial energy-efficiencies in the microwave-assisted vacuum preparation of polyphosphoric acid Chemical Engineering and Processing: Process Intensification 50 (2011) 1193-1197. 